\title{
Analysis of Microscopic Examination of Pulled Out Hair in Telogen Effluvium Patients
}

\author{
So Hee Park, Jung Eun Seol, Do Hyeong Kim, Hyojin Kim \\ Department of Dermatology, Busan Paik Hospital, Inje University College of Medicine, Busan, Korea
}

Background: Telogen effluvium (TE) is characterized by massive hair shedding and results from an abrupt conversion of anagen to telogen. Differentiation of TE with other hair disorders showing massive hair shedding is difficult. Objective: To evaluate the morphologic characteristics of pulled out hair of TE. Methods: We analyzed microscopic examination of pulled out hairs by hair pull test in TE patients, and compared these hairs with pulled out hairs in diffuse alopecia areata (AA) patients and healthy volunteers. Results: Hair microscopic examination in 44 TE patients (370 hairs) and 30 healthy volunteers (295 hairs) were as follows: typical club hair, 32.7\%, 62.7\%; club hair with tail, 23.5\%, 23.7\%; club hair with remnant sheath, $23.0 \%, 6.8 \%$; club hair with both tail and remnant sheath, $18.9 \%, 5.4 \%$; and unclassified hair, $1.9 \%, 1.4 \%$. The examination in 7 diffuse AA patients $(63$ hairs) was as follows: typical club hair, $23.9 \%$; atypical club hair, $47.7 \%$; and distinctive AA hair, $28.4 \%$. There was statistical difference between TE patients and healthy volunteers group ( $p=0.000$ ). Conclusion: Increased atypical club hair in microscopic hair examination might be characteristics of TE. (Ann Dermatol 32(2) 141 145, 2020)

\section{-Keywords-}

Alopecia, Alopecia areata, Hair

Received October 21, 2019, Revised November 22, 2019, Accepted for publication December 7, 2019

Corresponding author: Hyojin Kim, Department of Dermatology, Busan Paik Hospital, Inje University College of Medicine, 75 Bokji-ro, Busanjin-gu, Busan 47392, Korea. Tel: 82-51-890-6135, Fax: 82-51-897-6391, E-mail: derma09@hanmail.net

ORCID: https://orcid.org/0000-0003-0987-4938

This is an Open Access article distributed under the terms of the Creative Commons Attribution Non-Commercial License (http://creativecommons. org/licenses/by-nc/4.0) which permits unrestricted non-commercial use, distribution, and reproduction in any medium, provided the original work is properly cited.

Copyright $\odot$ The Korean Dermatological Association and The Korean Society for Investigative Dermatology

\section{INTRODUCTION}

Telogen effluvium (TE) is caused by an abrupt conversion of anagen hairs to telogen hairs on the scalp irrespective of the cause ${ }^{1,2}$. TE is diagnosed quantitatively, and presents with massive, diffuse hair shedding on scalp (more than 100 telogen hairs per day) ${ }^{3}$. It generally occurs about 3 months after a major illness or other stress (surgery, childbirth, rapid weight loss, nutritional deficiency, high fever or massive hemorrhage), medication or thyroid dysfunction $^{2}$. There are many relevant investigations for diagnosis of TE, which include hair loss count, hair pull test (HPT), hair pluck test, unit area trichogram, phototrichogram, histopathology, dermatoscopy, and laboratory test (complete blood count, serum ferritin, serum thyroid stimulating hormone, etc. $)^{1}$. However, differentiation of TE with other hair disorders showing massive hair shedding is still difficult. HPT is quick, noninvasive clinical examinations to monitor hair loss disorders ${ }^{4}$. To perform this test, the clinician selects 50 to 60 hairs and holds the bundle close to the scalp between the thumb, index finger, and long finger ${ }^{5}$. The clinician then firmly pulls on the bundle using slow traction as the fingers slide down the hair shaft ${ }^{5}$. If more than $10 \%$ of the hairs in each bundle are removed from a scalp area, the HPT is considered positive ${ }^{6}$.

There is no investigation about morphologic characteristics of pulled out hairs in TE patients. Hair shaft microscopic examination is useful diagnostic examination for hair loss diseases. Characteristics of hair in alopecia areata (AA) are well-known and helpful for diagnosis. However, those in TE is not well established. Herein, we investigated the morphologic characteristics of pulled out hairs in TE. 


\section{MATERIALS AND METHODS}

This study enrolled TE and diffuse AA patients from June 2013 to July 2015 in the Department of Dermatology in Busan Paik Hospital. TE met following criteria: (1) history of increased hair shedding; (2) absence of hair loss patch at initial and follow up visit; and (3) no past history of AA. The following criteria were applied to diagnose diffuse AA: (1) history of increased hair shedding; and (2) absence of hair loss patches at initial visit with occurrence of them in follow-up visits. Pulled-out hairs were harvested by gentle hair pulling using thumb and index finger. This was performed by one investigator.

Healthy volunteers who had neither hair loss disorders nor scalp disease were enrolled. In our study, around 10 hairs were obtained by gentle hair pulling performed by each subjects. Microscopic examination of pulled out hair performed in TE patients, diffuse AA patients and healthy volunteers. This study was approved by the institutional review board of the Inje University Busan Paik Hospital (approval no. 15-0270) and received consent form from all participants.

Analysis of morphologic characteristics of pulled out hair was based on classification of hairs into three categories; typical club hair, atypical club hair and distinctive AA hair. Typical club hair was defined as bulbous enlarge- ment of proximal hair root without following feature of atypical club hair or distinctive AA hair (Fig. 1A). Atypical club hair was subcategorized into club hair with tail, club hair with remnant sheath, club hair with both tail and remnant sheath and unclassified hair. Club hair with tail was defined as typical club hair with linear structure attached on proximal hair tip. Club hair with remnant sheath was defined as typical club hair with sheath surrounding proximal hair shaft (Fig. 1B D). Distinctive AA hair was defined as hair with at least one of following characterestics; tapering of proximal hair shaft, breakage of proximal hair shaft and hair with surface undulation (Fig. 2).

Morphologic characteristics of pulled out hairs in TE patients were compared with that of diffuse AA patients and healthy volunteers. In TE group, further comparative analysis was performed according to concurrent androgenetic alopecia (AGA) and degree of shedding based on the number of pulled out hair (TE $\geq 10$ vs. TE $<10$ ). Comparison between initial visit and follow-up visit was also performed.

Pearson's chi-square test and Fisher's exact test were used for statistical analysis of morphologic characteristics of pulled out hairs. Results were considered statistically significant at a $p<0.05$.
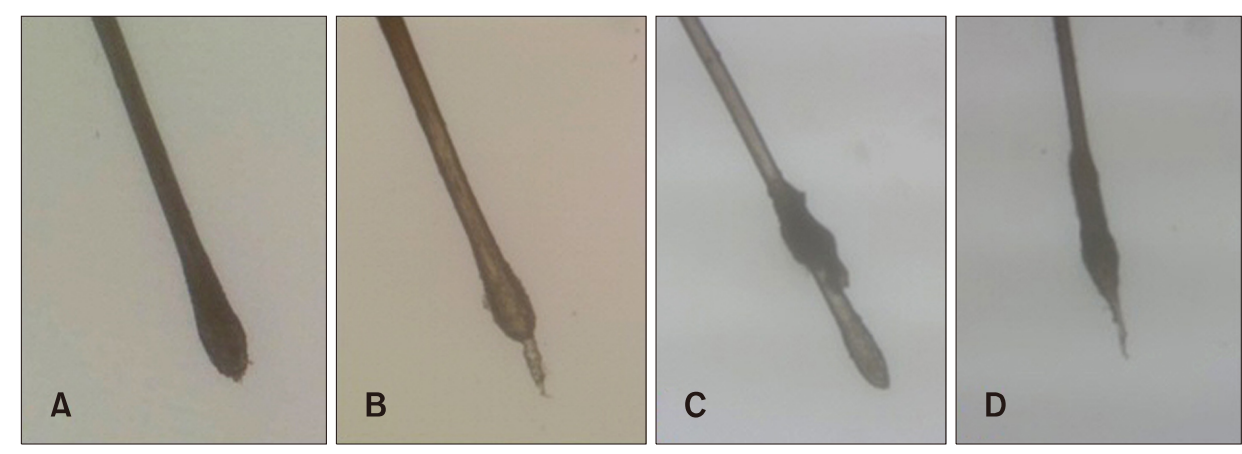

Fig. 1. Pulled out hairs were classified microscopically by morphology into typical club hair (A), club hair with tail (B), club hair with remnant sheath $(C)$, and club hair with both tail and remnant sheath (D) (A $\sim$ D: $\times 40$ ).
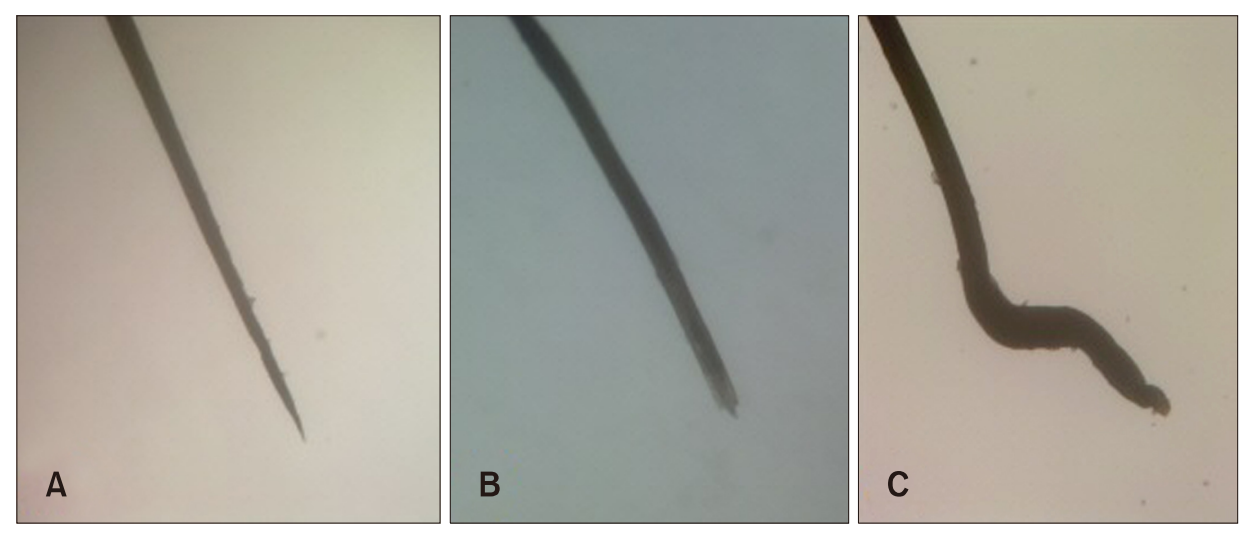

Fig. 2. Distinctive AA hairs include hair with tapering of proximal hair shaft (A), hair with breakage of proximal hair shaft (B) and hair with surface undulation $(C)(A \sim C: \times 40)$. 


\section{RESULTS}

\section{Demographic and clinical information of subjects}

Forty-four TE patients were enrolled in this study ( 3 males and 41 females) with a mean age of 54.4 years (range, 9 82). Most common etiology was idiopathic (38.6\%), followed by major illness (25.0\%). Fourteen subjects (31.8\%) had concomitant AGA. The characteristics of TE patients

Table 1. Demographic and clinical information of telogen effluvium patients

\begin{tabular}{lrl}
\hline \multirow{2}{*}{ Characteristic } & \multicolumn{2}{c}{ Subjects $(\mathrm{n}=44)$} \\
\cline { 2 - 3 } & $\mathrm{n}(\%)$ & Mean (range) \\
\hline Sex & $3(6.8)$ & \\
$\quad$ Male & $41(93.2)$ & \\
$\quad$ Female & & $54.4 \quad(9 \sim 82)$ \\
Age & & \\
Alopecia & $30(68.2)$ & \\
TE only & $14(31.8)$ \\
TE with AGA & & \\
Trigger factors of TE & $17(38.6)$ \\
Idiopathic & $11(25.0)$ \\
Major illness & $7(15.9)$ \\
Drug & $4(9.1)$ \\
$\quad$ Emotional stress & $3(6.8)$ \\
Weight loss & $2(4.6)$ \\
Childbirth & \\
\hline
\end{tabular}

TE: telogen effluvium, AGA: androgenetic alopecia.

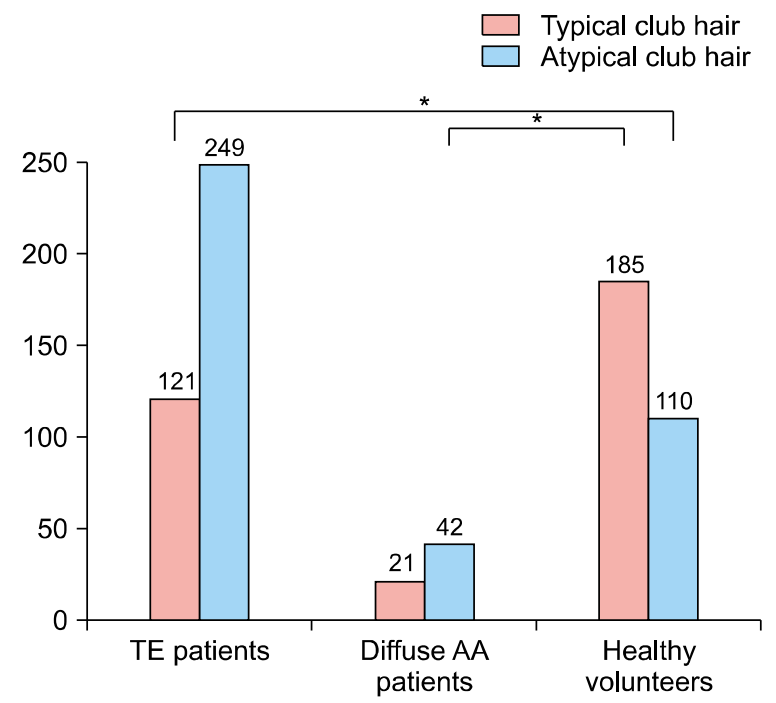

are shown in Table 1.

There were 7 enrolled patients with diffuse AA (4 males and 3 females) with a mean age of 39.7 years (range, 5 74) and 30 healthy volunteers (6 males and 24 females) with a mean age of 45.1 years (range, $22 \sim 82$ ).

\section{Microscopic examination of pulled-out hair in TE}

The results of morphologic evaluation of pulled out hairs are shown in Fig. 3. A total of 370 hairs in TE patients group were analyzed (2 to 35 hairs in each patient). Atypical club hair was $67.3 \%$, and club hair with tail was the most common. In atypical club hair, club hair with tail occupied highest proportion $(23.5 \%)$, followed by club hair with remnant sheath $(23.0 \%)$, club hair with both tail and remnant sheath $(18.9 \%)$, and unclassified hair $(1.9 \%)$.

In further analysis of TE $\geq 10$ group, the number of pulledout hair was from 11 to 35 (mean 19.6). The percentage of typical club hair was ranging $18.2 \% \sim 91.7 \%$. However, there was no significant statistical difference. The distribution of TE according to concurrent AGA was not significantly different (Table 2).

There were significance differences in distribution between pulled out hairs in initial visit and those of followup visits. The rate of typical club hair was higher in those of follow-up visit. In further analysis conducted on 18 subjects in whom both pulled-out hairs of initial visit and those of follow visit was obtained, there were statistical significance and the rate of typical club hair in initial visit

Fig. 3. Analysis of morphologic characteristics of pulled out hairs between 3 groups of telogen effluvium patients, diffuse alopecia areata patients, and healthy volunteers $\left({ }^{*} p<0.05\right)$. Distinctive AA hairs were excluded in this analysis. TE: telogen effluvium, AA: alopecia areata. 
Table 2. Analysis of morphologic characteristics of pulled out hairs

\begin{tabular}{|c|c|c|c|c|c|c|}
\hline Variable & $\begin{array}{l}\text { Shedding } \\
<10(\mathrm{n}=33)\end{array}$ & $\begin{array}{l}\text { Shedding } \\
\geq 10 \quad(n=11)\end{array}$ & $\begin{array}{l}\text { TE without AGA } \\
\text { patients }(n=30)\end{array}$ & $\begin{array}{l}\text { TE with AGA } \\
\text { patients }(n=14)\end{array}$ & Initial visit & $\begin{array}{l}\text { Follow-up } \\
\text { visits }\end{array}$ \\
\hline Typical club hair & $54(35.1)$ & $67(31.0)$ & $87(31.1)$ & $34(37.8)$ & $13(19.4)$ & $72(37.7)$ \\
\hline Atypical club hair & $100(64.9)$ & $149(69.0)$ & $193(68.9)$ & $56(62.2)$ & $54(80.6)$ & $119(62.3)$ \\
\hline Statistical analysis* & \multicolumn{2}{|c|}{ No statistical difference $(p=0.413)$} & \multicolumn{2}{|c|}{ No statistical difference $(p=0.238)$} & \multicolumn{2}{|c|}{ Statistical difference $(p=0.006)$} \\
\hline Typical club hair & $54(35.1)$ & $67(31.0)$ & $87(31.1)$ & $34(37.8)$ & $13(19.4)$ & $72(37.7)$ \\
\hline Club hair with tail & $32(20.8)$ & $55(25.5)$ & $65(23.2)$ & $22(24.4)$ & $19(28.4)$ & $46(24.1)$ \\
\hline Club hair with remnant sheath & $36(23.4)$ & $49(22.7)$ & $68(24.3)$ & 17 (18.9) & $13(19.4)$ & $44(23.0)$ \\
\hline Club hair with tail and remnant sheath & $31(20.1)$ & $39(18.1)$ & $54(19.3)$ & $16(17.8)$ & $19(28.4)$ & $26(13.6)$ \\
\hline Unclassified hair & $1(0.6)$ & $6(2.7)$ & $6(2.1)$ & $1(1.1)$ & $3(4.5)$ & $3(1.6)$ \\
\hline Statistical analysis ${ }^{\dagger}$ & \multicolumn{2}{|c|}{ No statistical difference $(p=0.453)$} & \multicolumn{2}{|c|}{ No statistical difference $(p=0.679)$} & \multicolumn{2}{|c|}{ Statistical difference $(p=0.001)$} \\
\hline
\end{tabular}

Values are presented as number (\%). We divided groups by (1) TE according to degree of shedding, (2) telogen effluvium patients with and without androgenetic alopecia, and (3) initial visit and those of follow-up visits. Pulled out hairs were evaluated with i) 'typical club hair' vs. 'atypical club hair', ii) 'typical club hair' vs. 'club hair with tail' vs. 'club hair with remnant sheath' vs. 'club hair with tail and remnant sheath' vs .'unclassified hair' respectively. AGA: androgenetic alopecia, TE: telogen effluvium. *The comparison was analyzed by chi-square analysis. ${ }^{\dagger}$ The comparison was analyzed by linear by linear analysis.

was much lower (Table 2).

\section{Microscopic examination of pulled-out hair in diffuse AA patients and healthy volunteers}

In 7 patients diagnosed as diffuse AA (total 63 hairs, 2 to 31 hairs in each patient), atypical club hair was the most common $(47.7 \%)$, however distinctive AA hair $(28.4 \%)$ was also observed considerably in amount. The distribution of distinctive AA hair was as follow: tapering of proximal hair shaft $(21.6 \%)$, breakage of proximal hair shaft $(3.4 \%)$ and hair with surface undulation $(3.4 \%)$.

In 30 healthy volunteers (total 295 hairs, 8 to 10 in each subject), typical club hair was the most common (62.7\%). Among atypical club hair, club hair with tail was the most common in this group.

\section{Comparison of distribution of microscopic examination in three groups}

Mean number of pulled out hair was 8.4, 12.6, 9.8 in TE, diffuse AA patients and healthy volunteer respectively $(p>0.05)$. Also, we analyzed the distribution of typical club hair and atypical club hair in three group (distinctive AA hairs in diffuse AA patients such as 'dystrophic hair', 'dysplastic hair', and 'broken hair' were excluded in this analysis). There was statistical difference in hair morphology among 3 groups in distributions of whole atypical club hair as well as its subclassification in those of subcategorization. Post-hoc comparison between each 2 groups also showed statistically significant difference with one exception of distribution of whole atypical club hair between TE and diffuse AA (Fig. 3).

\section{DISCUSSION}

Approximately $86 \%$ of scalp hairs are anagen hair, $1 \%$ are catagen hair, and $13 \%$ are telogen hair in physiologic conditions ${ }^{1}$. The anagen phase lasts for 3 to 7 years, and is followed by catagen for 2 weeks. In catagen phase, protein and pigment production is stopped, involution of hair follicles begins, and reorganization of extracellular matrix occurs $^{7}$. In telogen phase, hair follicles involute reaching $<50 \%$ of its anagen size. Hair does not fall out promptly in telogen phase, but rather stays in hair follicles for a while'. Recently the term 'exogen' has been used to describe the timing of hair falling. It is suggested that there will be many processes before hair falling in telogen phase, such as loss of desmoglein 3 (acting as anchoring protein of telogen hair) ${ }^{7,8}$. Morphologically, remaining parts in telogen phase is a peg of epithelial cells above the resting dermal papilla ${ }^{7}$.

Healthy people lose up to 100 hairs a day ${ }^{9}$. However, in TE patients, massive shift of anagen to telogen develops abruptly, and the ratio of anagen to telogen changes to $70: 30^{2}$. TE is characterized by excessive shedding of scalp hair, sometimes loss of more than 300 hairs per day, although it may occur in AA or other hair loss disease $e^{2,3,10,11}$. The analysis of this study was based on morphologic characteristics. Significance of each atypical club hair was not evident and elucidation of each in hair cycle was not performed. However based on previous study, when interpretating tail as epithelial column, it could be presumed that club hair with tail was compatible with late catagen or early telogen hair according to the length ${ }^{12}$. As for club hair with sheath, remnant sheath might be considered as remaining inner root sheath or outer root sheath of telogen 
hair $^{12}$. Accordingly, club hair with both tail and sheath might be in transition from catagen to telogen.

Amount of hair shedding in physiologic state might influenced by intrinsic cohesive force between hair shaft and surrounding tissue which is determined by stage of hair cycle and intrinsic characteristics of each individual such as age, and degree and frequency of exposure of pulling power, such as hair combing or shampooing ${ }^{6,13-15}$. In this study, healthy volunteers showed diverse morphology in hair microscopic examination, which was not accordant with our assumption that almost shed hair would be typical club hair. Club hair with tail was the most common among atypical club hair. It is probable that subject with hair loss disease in subclinical stage might be included in healthy volunteer.

The typical club hair-atypical club hair ratio was reversed in TE compared with that of healthy volunteer. This result indicated that alteration of physiologic hair cycle resulting in qualitative as well as quantitative change. It could be supported by the distribution of the percentage of typical club hair in each subject in severe TE and healthy volunteer. In healthy volunteer, subjects with greater than $50 \%$ in typical club hair ratio was 21 out of $30(20 \% \sim 100 \%)$, whereas in 11 subjects in TE $\geq 10$ group, only one showed greater than $50 \%$ in typical club hair ratio $(8.3 \% \sim 81.8 \%)$. These results might suggest that TE is related to discordance with surrounding tissue resulting morphologic change as well as changes in duration of hair cycle. Distribution in atypical club hair was also changed; increased club hair with remnant sheath and club hair with both tail and sheath. Although TE with concurrent AGA was not significantly different from TE without AGA in this study, further evaluation might be necessary considering TE results from diverse pathogenesis. In addition, increased in the rate of typical club hair in follow-up visit compared with those in initial visit might suggest reversal of the rate of typical club hair could be early change in recovery of TE followed by cessation of hair shedding.

This study is significant in that it identified the qualitative changes of pulled out hair in TE, which might be applied in diagnosing TE directly instead of diagnosis by exclusion of other causes of increased hair shedding. Morphologic evaluation of pulled out hairs might be helpful in diagnosing TE. Further research on the hair cycling and shed hair morphology in physiologic state, and interpretation of each morphology will be necessary. Lack of standardization in shampooing schedule of subject and pulling power in healthy volunteer could be limitation.

\section{CONFLICTS OF INTEREST}

The authors have nothing to disclose.

\section{ORCID}

So Hee Park, https://orcid.org/0000-0002-9600-799X

Jung Eun Seol, https://orcid.org/0000-0002-3029-9635

Do Hyeong Kim, https://orcid.org/0000-0001-6148-8810

Hyojin Kim, https://orcid.org/0000-0003-0987-4938

\section{REFERENCES}

1. Grover C, Khurana A. Telogen effluvium. Indian J Dermatol Venereol Leprol 2013;79:591-603.

2. Shapiro J. Clinical practice. Hair loss in women. N Engl J Med 2007;357:1620-1630.

3. Mubki T, Rudnicka L, Olszewska M, Shapiro J. Evaluation and diagnosis of the hair loss patient: part I. History and clinical examination. J Am Acad Dermatol 2014;71:415.e1415.e15.

4. McDonald KA, Shelley AJ, Colantonio S, Beecker J. Hair pull test: evidence-based update and revision of guidelines. J Am Acad Dermatol 2017;76:472-477.

5. Hillmann K, Blume-Peytavi U. Diagnosis of hair disorders. Semin Cutan Med Surg 2009;28:33-38.

6. Shapiro J, Wiseman M, Lui H. Practical management of hair loss. Can Fam Physician 2000;46:1469-1477.

7. McElwee KJ, Sinclair R. Hair physiology and its disorders. Drug Discov Today Dis Mech 2008;5:e163-e171.

8. Hardy MH. The secret life of the hair follicle. Trends Genet 1992;8:55-61.

9. Jackson AJ, Price VH. How to diagnose hair loss. Dermatol Clin 2013;31:21-28.

10. Alkhalifah A, Alsantali A, Wang E, McElwee KJ, Shapiro J. Alopecia areata update: part I. Clinical picture, histopathology, and pathogenesis. J Am Acad Dermatol 2010;62:177-188; quiz 189-190.

11. Otberg N. Primary cicatricial alopecias. Dermatol Clin 2013; 31:155-166.

12. Van Neste D, Leroy T, Conil S. Exogen hair characterization in human scalp. Skin Res Technol 2007;13:436-443.

13. Hoffmann R. TrichoScan: combining epiluminescence microscopy with digital image analysis for the measurement of hair growth in vivo. Eur J Dermatol 2001;11:362-368.

14. Piérard GE, Piérard-Franchimont C, Marks R, Elsner P; EEMCO group (European Expert Group on Efficacy Measurement of Cosmetics and other Topical Products). EEMCO guidance for the assessment of hair shedding and alopecia. Skin Pharmacol Physiol 2004;17:98-110.

15. Van Neste MD. Assessment of hair loss: clinical relevance of hair growth evaluation methods. Clin Exp Dermatol 2002; 27:358-365. 